\title{
Clinical Recommendations of DEGRO and AGO on Preferred Standard Palliative Radiotherapy of Bone and Cerebral Metastases, Metastatic Spinal Cord Compression, and Leptomeningeal Carcinomatosis in Breast Cancer
}

\author{
Rainer Souchon ${ }^{a *}$ Petra Feyer $^{\mathrm{b} *}$ Christoph Thomssen ${ }^{\mathrm{c} * *}$ Tanja Fehm $^{\mathrm{d} * *}$ \\ Ingo Diel ${ }^{* * *} \quad$ Ulrike Nitz ${ }^{f * *}$ Wolfgang Janni ${ }^{* *}$ Joachim Bischoff ${ }^{\text {h**}}$ Rolf Sauer ${ }^{i *}$
}

${ }^{a}$ Department of Radiation Oncology, University of Tübingen, 'bepartment of Radiation Oncology, Klinikum Neukölln, Berlin, ${ }^{\mathrm{c} D e p a r t m e n t}$ of Gynecology, University of Halle (Saale), 'Department of Obstetrics and Gynecology, University of Tübingen, ${ }^{e}$ Center for Holistic Gynecology, Mannheim, ${ }^{\mathrm{f} B r u s t z e n t r u m ~ N i e d e r r h e i n, ~ M o ̈ n c h e n g l a d b a c h, ~}{ }^{9}$ Department of Obstetrics and Gynecology, University of Düsseldorf, hepartment of Obstetrics and Gynecology, University of Magdeburg, 'Department of Radiation Oncology, University of Erlangen, Germany

\section{Keywords}

Metastatic breast cancer . Palliative radiation therapy . Bone metastasis · Cerebral metastasis · Metastatic spinal cord compression - Leptomeningeal carcinomatosis

\section{Summary}

Background: To provide guidance for clinical practice on preferred standard palliative radiotherapy (RT) of different sites of metastasis for breast cancer patients based on current published evidence complemented by expert opinion. Methods: The breast cancer expert panel of the German Society for Radiation Oncology (DEGRO) and members of the Working Party of Gynecologic Oncology (AGO) Breast Committee formulated recommendations based on the panel's interpretation of the level of evidence referring to the criteria of evidence-based medicine added to the AGO grades of recommendation. Results: For different types and sites of metastasis, distinct therapeutic goals (alleviation of symptoms, pain relief, local tumor control, prevention or improvement of neurological deficits, stabilization of the spine or other bones) require complex approaches considering individual factors (i.e. life expectancy, tumor progression at other sites). With regard to different therapeutic goals, different dose concepts and fractionation schedules, and single- versus multi-fraction palliative RT should be adapted individually. Conclusions: RT is an effective tool in palliation treatment of bone metastasis (BM), cerebral metastasis (CM) and metastatic spinal cord compression (MSCC), or leptomeningeal carcinomatosis (LC) and plays a central role in an interdisciplinary approach. Preferred technique, targeting, and different dose schedules are described in detail in the DEGRO guidelines, which are also integrated in the updated 2010 AGO recommendations.

*For the breast cancer expert panel of the German Society for Radiation Oncology (DEGRO).

**For the Working Party of Gynecologic Oncology (AGO) Breast Committee.

\section{Schlüsselwörter}

Mammakarzinom, metastasiertes · Radiotherapie, palliative $\cdot$ Metastasen, ossäre $\cdot$ Metastasen, zerebrale · Metastatisches spinales Kompressionssyndrom . Leptomeningeosis carcinomatosa

\section{Zusammenfassung}

Hintergrund: Erstellung praktischer Handlungsempfehlungen zur palliativen Radiotherapie (RT) verschiedener Metastasierungsmanifestationen bei Patientinnen mit Mammakarzinom auf Basis aktuell publizierter Evidenz und ergänzender Expertenmeinung. Methodik: Die vom Expertengremium der Organgruppe Mamma der Deutschen Gesellschaft für Radioonkologie (DEGRO) und Mitgliedern der Kommission Mamma in der Arbeitsgemeinschaft Gynäkologische Onkologie (AGO) erstellten Empfehlungen basieren auf der Analyse kontrollierter klinischer Studien und internationalen sowie nationalen praktischen Handlungsanleitungen und den Interpretationen der Evidenzgrade nach Kriterien der Evidenz-basierten Medizin, ergänzt um die Empfehlungsgrade der AGO-Kommission. Ergebnisse: Unterschiedliche Therapieziele für die Palliation (Schmerzlinderung, lokale Tumorkontrolle, Prävention und Rückbildung neurologischer Ausfälle, ossäre Stabilisierung) erfordern jeweils auf den individuellen Fall abgestimmte, differenzierte, radioonkologische Behandlungskonzepte unter Berücksichtigung von Lebenserwartung und Tumoraktivität an den verschiedenen Manifestationsorten. Je nach Palliationsziel kommen unterschiedliche Dosiskonzepte und Fraktionierungsschemata in Form von Einzel- oder Multifraktionierungs-RT zur Anwendung. Schlussfolgerungen: Die RT ist ein effektiver Bestandteil bei der palliativen Behandlung bei ossärer (BM) oder zerebraler (CM) Metastasierung, des tumorbedingten spinalen Kompressionssyndroms (MSCC) oder bei Leptomeningeosis carcinomatosa (LC). Techniken, Zielvolumenbestimmungen und verschiedene Dosiskonzepte für unterschiedliche klinische Konstellationen werden in den DEGRO-Handlungsempfehlungen, die in die aktualisierten AGO-Empfehlungen 2010 integriert wurden, detailliert beschrieben.

\begin{tabular}{ll}
\hline KARGER & @ 2010 S. Karger GmbH, Freiburg \\
Fax +497614520714 & Accessible online at: \\
Information@Karger.de & www.karger.com/brc \\
www.karger.com &
\end{tabular}




\section{Introduction}

Pathologic fractures due to bone metastasis (BM) and metastatic spinal cord compression (MSCC) or symptomatic cerebral metastasis (CM) lead to a pronounced deterioration of the quality of life and are oncologic emergencies asking for a rapid onset of therapy. The potential for long-lasting and severe complications necessitate an immediate and precise diagnostic workup and an effective, multimodal palliative therapy. Radiation therapy (RT) plays a central role in this process due to the radiosensitivity of breast cancer cells.

The breast cancer expert panel of the German Society for Radiation Oncology (DEGRO) has developed guidelines for palliative RT, with special emphasis on the peculiarities of metastatic breast cancer in synopsis with multimodal concepts $[1,2]$. These updates include recently published data on surgical and radiotherapeutic approaches with respect to aspects of fractionation, novel techniques, and developments in target volume definition.

About $20 \%$ of patients with breast cancer suffer from BM. Skeletal involvement is present in more than half of the cases with distant metastases. Less than $10 \%$ of the patients with bone metastases will develop MSCC, usually late in the course of the disease. MSCC is in more than $80 \%$ secondary to bone metastases to the vertebral column, associated with a mechanical compression of the myelon. Intraspinal metastases with epi- or intradural localization can be found in rare cases.

Breast cancer is the second most common cause for brain metastases $(\mathrm{CM})$, occurring with increasing incidence, i.e. in $10-40 \%$ of patients who develop distant disease; parenchymal metastases are found at autopsy in 30-40\%, and leptomeningeal central nervous system (CNS) metastases in 5-16\%. Risk factors are young age, negative estrogen receptor status, poor differentiation, Her2 overexpression, and basal cell type. LC is less common, with $1-5 \%$ of metastasized patients; half of these patients suffer from simultaneous CM.

\section{Bone Metastasis and Metastatic Spinal Cord Compression}

\section{Clinical Presentation, Prognostic Factors, and Diagnostic Workup}

The signs and symptoms of BM largely depend on the location and the mechanical stress on the affected parts of the bone. It manifests itself in pain, movement restrictions, fractures and/or neurological symptoms. For patients with breast cancer, the occurrence of these symptoms is suggestive of bone metastases until proven otherwise. Bone scintigraphy is a widely used method for the diagnostic workup, allowing a total skeletal assessment, which is helpful in determining the extent of the metastatic spread. Further diagnostic procedures are usually necessary due to the lack of specificity: Conventional X-ray imaging is used for the evaluation of suspiciously enhancing lesions in the extremities. It allows a differentiation between lytic, blastic, or mixed lytic-blastic osseous metastases, provided a sufficiently high proportion $(>30 \%)$ of the bone matrix is destroyed. Computed tomography (CT) in high resolution mode shows also smaller osteolysis. Moreover, tumor extension into adjacent soft tissues and the stability of the bone are better delineated with $\mathrm{CT}$ than with conventional X-rays.

MSCC is usually preceded by week-long back pain with increasing motion- and load-dependent intensity. Sensitivity disorders and/or muscle weakness in the caudally located body parts and consecutive mobility impairments (ataxia) as well as dysfunction of the bladder and the anal sphincter build the typical clinical evidence of MSCC. Significant prognostic factors are the degree of mobility before RT (ambulatory vs. bedridden) and the interval between onset of symptoms and initiation of RT (LoE 2b, B). Duration, temporal development, and extent of neurological deficits are also of prognostic significance for MSCC, which is reflected in the different prognostic scores (LoE 2b, B). For the diagnostic workup of MSCC, it is mandatory to assess a specific history of the current clinical situation and a clinical neurological examination for estimation of the level and the extent of the metastatic process in the spinal canal, thus enabling targeted imaging studies: magnetic resonance imaging (MRI; exploring extent, intradural/extradural vs. intraspinal masses), CT (assessing stability and extent of destruction) and - optional - conventional X-rays (getting further information regarding the extent of deformity and stability).

\section{Goals of Therapy}

Diagnostic workup and treatment decisions are individualized and oriented on the respective therapy goal:

- reduction of pain

- local tumor control

- restabilization of the skeleton

- prevention or control of neurologic deficits

- improvement of palliative care situation

In respect of the chief complaints and imminent complications, different goals of therapy may be defined. An interdisciplinary consensus is recommended to prioritize the respective goal.

Especially in case of patients with MSCC from metastasizing breast cancer, the aim of therapy should be defined according to the neurologic situation, the time course of neurologic deficits, and the initial effect of high-dose steroids. Mobility and the capability of self-care are of central importance for the quality of life of the patient. 


\section{(Radio-)Therapeutic Options}

Due to protracted tumor response, it is recommended to continue the pain medication during the initial phase of the fractionated RT. Although a rapid pain reduction within $24-48 \mathrm{~h}$ might be observed in some patients - presumably due to radiation-induced cell death of tumor-infiltrating leukocytes a substantial reduction of the tumor volume, allowing a relevant tapering of the pain medication, will not be seen before 7-8 days after the start of RT. Pain medication should be initiated according to the World Health Organization (WHO) scheme (by the ladder, by the clock, by the mouth). A combination of peripherally and centrally acting medication should be given, starting with non-opioids \pm co-analgetics (level I), which may be combined with weakly (level II) or strongly acting opioids (level III).

The indication for a surgical intervention before RT depends on the stability of the bone and the prognosis of the patient. It should be given more generously in lesions of the weight-bearing lower extremities. However, the lack of a validated set of criteria to determine bony and in particular spinal instability makes patient selection for surgical intervention difficult. Postoperative RT is given following surgical stabilization, to prevent progression of the bony destruction and to improve remineralization. Because surgical resection of bone metastases is virtually never complete, postoperative RT is required to provide local tumor control. This also applies to vertebroplastic interventions.

Immediate ( $<24 \mathrm{~h}$, max. $<48 \mathrm{~h})$ surgical decompression with tumor debulking followed by RT is the method of choice in case of vertebral column instability, vertebral compression,

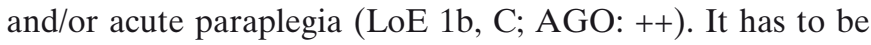
considered that laminectomy additionally destabilizes the vertebral column.

\section{Emergency Measures in MSCC}

The initial intervention should be an intravenous (i.v.) application of high-dose steroids, up to doses of $96 \mathrm{mg} /$ day. A potential remission of neurological symptoms can be expected as early as 4-6 h post administration. The extent of the initial remission is predictive for the effect of RT and therefore guides the further therapeutic steps (LoE 3b, C; AGO: ++ [3]).

\section{Bisphosphonates}

Bisphosphonates are potent inhibitors of osteoclasts. They are indicated in case of bone metastases in order to reduce metastases-related skeletal events (fractures, pain attacks, etc.). Additionally, there is evidence that bisphosphonates improve recalcification after RT and also have antineoplastic potential (LoE 1a, A; AGO: ++).

\section{Radiotherapy}

$\mathrm{RT}$ is indicated for bone metastases from breast cancer in the following situations:

- imminent fracture (LoE 1a, B; AGO: ++)

- bone pain (LoE 1a, B; AGO: ++)

- neuropathic bone pain (LoE 1b, B; AGO: ++)

- asymptomatic isolated bone metastases (LoE 5, D; AGO: \pm )

- after surgical intervention (LoE 1a, B)

Specifically in the case of MSCC:

- MSCC without neurologic deficits (LoE 1b, C; AGO: ++)

- beginning paraplegia (also after initial response to steroids) (LoE 1b, C; AGO: ++)

- inoperability (LoE 1c)

- postoperative, after any surgical intervention ( $\mathrm{LoE} 1 \mathrm{c}, \mathrm{B})$

RT is the method of choice in inoperable cases due to:

- Low rates of side effects

- short hospitalization/outpatient procedure

- radiosensitivity of breast cancer

These advantages are also relevant in patients with a dismal overall prognosis, short life expectancy, and severe comorbidity, especially because RT is equivalent to simple laminectomy with respect to the functional outcome.

Local RT is indicated postoperatively to achieve local tumor control and should be initiated as soon as possible (LoE 2a, A; AGO:++).

\section{Technical Aspects of RT}

\section{Target Volume}

The definition of the clinical target volume (CTV) is not based on scientific data, but rather on clinical experience. The CTV includes the affected part of the bone with an adequate (e.g. 1-2 cm) and anatomically reasonable safety margin and, if present, a soft tissue component. In case of vertebral involvement, the CTV should always include the complete vertebral body (LoE V). In the case of unaffected compacta, extension of the CTV beyond the compacta is not recommended.

In clinically suspected intradural extension, MRI is recommended to delineate the meningeal enhancement and include it into the CTV.

Metal components used for stabilization should be included completely in the radiation volume when tumor cell dissemination due to the surgical procedure is suspected.

\section{Technique and Dose Prescription}

Technique, dose prescription and fractionation should be adjusted to the therapeutic goal, the prognosis of the patient, and localization and size of the target volume.

General recommendations are:

- Patients with metastases benefit from modern treatment planning techniques because the target volume is restricted to the metastasis and a prophylactic irradiation of adjacent 
tissue is not necessary. Modern treatment planning methods help conform the isodoses to the shape of the target volume, especially when chemotherapy is given or when the patient is in a limited overall condition.

- For patients in reduced overall condition and pain, complex irradiation techniques are, in general, not of increased value due to prolonged treatment times as well as higher needs for precise positioning and immobilization. Simple techniques with short daily treatment times should be used when analgesia is the main goal of treatment.

- Bony lesions of the extremities can be treated with simple techniques, e.g. opposed portals.

- Three-dimensional (3D) treatment planning is recommended in the case of a relevant soft tissue component, especially for lesions of the trunk.

The meta-analysis by Sze et al. [4], including 3435 patients with bone metastases, including 1350 breast cancer patients, demonstrated no difference in the analgetic effect of $1 \times 8$ Gy in comparison to $10 \times 3 \mathrm{~Gy}$. The rate of pathologic fractures and re-irradiation was markedly higher in the group with $1 \times 8$ Gy (table 1). Comparable results were demonstrated in the meta-analysis by Chow et al. [5] and in a recent prospective randomizd study by Foro Arnalot et al. [6].

\section{Special Technical Considerations and Dosing in MSCC}

In general, treatment planning is performed using CT or MRI for proper target volume definition. Different fractionation concepts have been evaluated in prospective studies $(1 \times 8$, $5 \times 4,10 \times 3,15 \times 2.5,20 \times 2$ Gy). However, no regimen proved to be markedly superior. Prolonged fractionation appears to be beneficial as to longer-lasting therapeutic effects and a lower rate of in-field recurrences (and necessity for re-irradiation). A dose escalation above $30 \mathrm{~Gy}(10 \times 3 \mathrm{~Gy})$ yielded no further advantages. Therefore, the appropriate fractionation should be chosen respecting the treatment duration, estimated life expectancy, and need for hospitalization.

\section{Re-Irradiation of Bone Metastases}

Indication and dosage of an optional re-irradiation depend on the previously applied dose, the initial response, the interval to the preceding RT, and the dose per fraction to critical organs. A re-irradiation of metastases in the extremities or the peripheral parts of the trunk is usually possible. In most cases, one may again apply full therapeutic doses, e.g. $10 \times 3$ Gy or $20 \times 2$ Gy, favoring the use of small single doses.

Prior to re-irradiation of the base of the skull, the vertebral column, and the pelvic bones, the overall radiation tolerance of critical organs like brain stem, spinal cord, bowel, and bladder has to be considered. 3D-conformal RT or intensitymodulated RT (IMRT) allows concave shaping of isodose distributions with at least partial sparing of critical structures adjacent to the target volume. In case of in-field recurrence of MSCC, re-irradiation can be performed in selected cases, again considering individual factors and therapeutic aims $(\operatorname{LoE} 3,0)$.

\section{Summary of Recommendations}

$\mathrm{BM}$ in breast cancer has a high clinical relevance and is commonly seen by the radiation oncologist. Therefore, it is necessary for the radiation oncologist to acquire in-depth knowledge and to offer a comprehensive management of this constellation.

The principles of therapy and the guidelines for palliative RT of BM and MSCC in patients with breast cancer are summarized in tables 2 and 3 .

\section{Supportive Care}

The early use of physical therapy and rehabilitation measures are of importance, in addition to medication (steroids, bisphosphonates, etc.).

Table 1. Effect of RT on BM (LoE 1a)

\begin{tabular}{lllllll}
\hline & \multicolumn{2}{l}{ Single-dose RT } & \multicolumn{3}{l}{ Fractionated RT } \\
\cline { 2 - 7 } & Sze [4] & Chow [5] & Foro Arnalot [6] & Sze [4] & Chow [5] & Foro Arnalot [6] \\
\hline Pain reduction, \% & 60 & 58 & 75 & 59 & 59 & 86 \\
Complete pain remission, \% & 34 & 23 & 15 & 32 & 24 & 13 \\
Re-irradiation, \% & 21.5 & 20 & 28 & 7.4 & 8 & 2 \\
Pathologic fractures, \% & 3 & 3.2 & not stated & 1.6 & 2.8 & not stated \\
\hline
\end{tabular}

Table 2. Guidelines for the treatment of BM

\begin{tabular}{ll}
\hline Therapeutic goal: pain reduction & single-dose RT $1 \times 8$ Gy (cave: $>8$ Gy to the myelon may cause paresis) (LoE 3) \\
Therapeutic goal: stabilization, good prognosis & fractionated regimen preferable, e.g. 10-12×3 Gy (LoE 2b) \\
Oligometastases & full dose-fractionated regimen recommended, e.g. 20-25×2 Gy to 40-50 Gy (LoE 2b) \\
\hline
\end{tabular}


Table 3. Guidelines for MSCC

\begin{tabular}{|c|c|}
\hline $\begin{array}{l}\text { Instability of vertebral column, bony compression } \\
\text { and/or paresis/paraplegia }\end{array}$ & immediate (within maximally $24-48 \mathrm{~h}$ ) surgical intervention and postoperative RT (LoE 2b) \\
\hline Spinal cord compression without neurologic deficits & $\begin{array}{l}\text { in ambulatory patients: RT (LoE } 2 \text { b); in case of analgesia as additional goal: short course of } \\
\text { RT with increased single doses; in case of remineralization as additional goal: fractionated } \\
\text { RT with conventional single doses }\end{array}$ \\
\hline Acute onset of paresis/paraplegia & surgical decompression followed by RT; RT when decompression is not possible (LoE 3) \\
\hline Inoperability & RT; choice of fractionation depending on life expectancy (LoE 3) \\
\hline After surgical decompression & $\mathrm{RT}(\mathrm{LoE} 2 \mathrm{~b})$ \\
\hline In case of (in-field) recurrence after previous RT & surgery (when possible); re-irradiation (using high-precision techniques) (LoE 4) \\
\hline
\end{tabular}

\section{Brain Metastases and Leptomeningeal Carcinomatosis}

$\mathrm{CM}$ is most commonly a late event occurring in the course of extensive metastatic disease. Patients with triple-negative and Her2-overexpressing tumors may present with $\mathrm{CM}$ as first site of recurrence or even oligometastatic cerebral disease.

\section{Clinical Presentation, Prognostic Factors, and Diagnostic Workup}

According to the anatomical site of the lesions, neurological symptoms of both CM and LC are headache, signs of intracranial pressure, motor weakness, seizures, cognitive deficits, and mental problems. Back pain following radicular patterns is common in LC with spinal involvement.

Generally, prognosis in both subgroups is dismal, with the Karnofsky performance score (KPS) being the most important prognostic parameter. Nevertheless, effective treatment may alleviate symptoms and occasionally prolong survival. For patients with $\mathrm{CM}$, even a survival of more than 3 years is achievable. In patients with LC, adequate therapy may prolong survival to more than 6 months and achieve a 1-year survival rate of up to $25 \%$.

For CM the Radiation Therapy Oncology Group (RTOG) defined three prognostic subgroups on the basis of data from 1200 patients, using the recursive partitioning analysis (RPA). Patients with breast cancer were adequately represented in these studies [7, 8].

- RPA class I: KPS $\geq 70 \%$, age $<65$ years, with controlled primary tumor and no extracranial metastases: expected median survival 11 months.

- RPA class III: KPS < 70\%: expected median survival 3 months.

- RPA class II: all others: expected median survival 6 months.

Prognostic factors for survival in multivariate analysis have been surgical resection, RPA classification, and no extracranial metastases. Other factors for predicting prognosis are the number of cerebral metastases (LoE $2 b$ ) and the response to steroid treatment.
In order to predict survival after whole-brain RT (WBRT), Rades et al. [9] established a new scoring system on the basis of a multivariate analysis of $>1000$ patients. The score refers to the following significant prognostic factors:

- age

- KPS

- extracranial tumor spread

- interval between tumor diagnosis and WBRT

For CM, the diagnostic procedure is oriented on clinical symptoms; most commonly, CT and MRI are used. If specific local treatment such as surgery or stereotactic RT is an option, MRI is superior to CT for delineation of metastases and detection of multiple lesions.

For differential diagnosis of LC, cerebrospinal fluid (CSF) cytology examination is mandatory, and the presence of malignant cells is evidentiary for LC. MRI of the complete craniospinal axis with gadolinium enhancement is the imaging technique of choice.

\section{Goals of Therapy}

Breast cancer death from $\mathrm{CM}$ is a rare event.

The aim of the treatment is to improve or stabilize the neurological status, maintain or regain quality of life, and, optimally, prolong survival ( $\mathrm{LoE} 2 \mathrm{~b}, \mathrm{~B}$; AGO: ++).

\section{Radiotherapeutic Options in CM}

In symptomatic patients, systemic application of corticosteroids is the standard treatment for brain edema. The optimal dose has been investigated in patients with primary brain tumors (LoE Ib). With dexamethasone, symptoms of intracranial pressure are rapidly alleviated within 4-24 h. A dose of $4 \mathrm{mg}$ is adequate to start with and may be increased according to remaining symptoms. However, corticoid application alone may not be sufficient with regard to a long-term effect (LoE 3a, B; AGO: \pm ).

The treatment choice for CM should be based on clinical predominant symptoms and verification in diagnostic imaging 


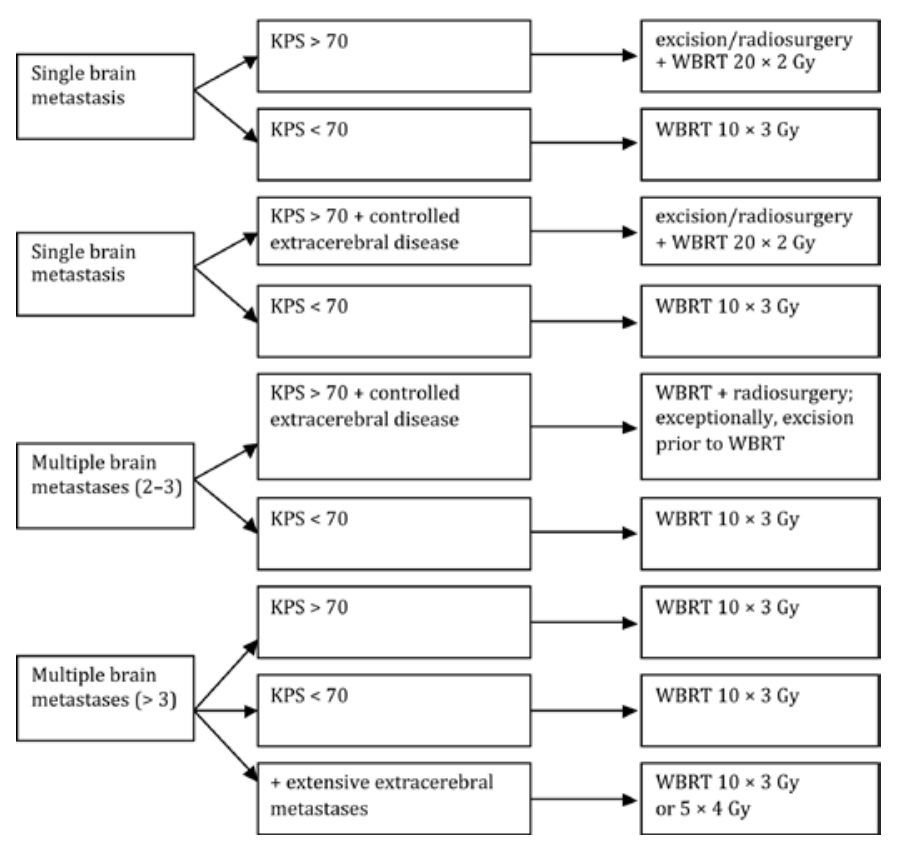

Fig. 1. Treatment algorithm for brain metastases.

as well as the number of brain metastases and the probability of extra-CNS tumor control. Together with the extent of the disease, the KPS determines whether excision or radiosurgery/stereotactic RT is feasible and if exclusive or additional WBRT is indicated (fig. 1).

In asymptomatic patients, the decision depends on the individual situation: for patients in RPA class I, specific treatment is mandatory, whereas in patients with RPA class III, symptomatic therapy may be preferable. In case of a long recurrence-free interval and lack of extracerebral tumor spread, histological verification should be considered prior to treatment.

In contrast to $\mathrm{CM}$, evidence-based guidelines for the treatment of LC are not available. Treatment of LC is preferentially multidisciplinary and mostly indicated when the diagnosis is unequivocal or symptoms are strongly suggestive in case of negative cytology.

\section{Solitary $C M$}

There are no randomized studies or data permitting a definite conclusion whether surgery and stereotactic RT are equally effective. Retrospective studies indicate that both treatments may achieve comparable results ( $\operatorname{LoE} 2 b$ ). Therefore, the treatment decision should be made individually regarding anatomic localization and size of the lesion, amenability for surgery, acute clinical risk, and the patient's preference.

Factors in favor of primary surgery are:

- histologic verification after a long recurrence-free interval

- need for immediate decompression in case of rapidly developing clinical deterioration or life-threatening symptoms
- tumor size $>3.5 \mathrm{~cm}$ and surgically favorable location

- surgery + WBRT + boost-stereotactic radiosurgery (SRS) (LoE 2a, B; AGO: ++ )

Factors in favor of primary RT (LoE $2 \mathrm{a})$ are:

- RPA class II

- no need for rapid decompression

- short recurrence-free interval

- no need for histologic verification due to unambiguous medical history (e.g. additional metastatic spread)

- high risk of surgery

- tumor location poorly amenable for surgery

WBRT following surgery, stereotactic RT or radiosurgery may improve the outcome of patients with a single brain metastasis who have favorable prognostic factors and a KPS of at least $70 \%$ without exctracranial tumor spread (RPA class I) or patients with RPA class II and metastases limited to the skeletal system. For patients with RPA class III, RT should be restricted to WBRT.

\section{Two or Three CM}

Resection or stereotactic RT may be considered (LoE 2b); additional WBRT further improves local tumor control. Immediate WBRT following excision or radiosurgery provides increased recurrence-free survival (LoE 1b). However, data do not permit a definite conclusion whether this translates into improved overall survival. Nonetheless, the aspect of improved quality of life justifies WBRT.

\section{WBRT of More than Three CM}

WBRT is the standard treatment (LoE 1a, A; AGO: ++). Several RTOG studies investigated different fractionation schedules (LoE 1b) and found a median survival time of 3-6 months, irrespective of fractionation. Remission rates and duration of response were comparable between subgroups treated with regimens of $50 \mathrm{~Gy} / 4 \mathrm{w}, 40 \mathrm{~Gy} / 3 \mathrm{w}, 40 \mathrm{~Gy} / 4 \mathrm{w}$, 30 Gy/2 w, 30 Gy/3 w, 20 Gy/1 w (LoE 2b, B; AGO: +). More hypofractionated regimens like $1 \times 10$ Gy or $2 \times 6$ Gy rapidly alleviate symptoms. However, the duration of this effect is short; therefore, these regimens are not recommended.

Several studies addressed the issue whether local dose escalation achieves improved survival. A boost up to $54.4 \mathrm{~Gy}$ did not yield better results ( $\mathrm{LoE} 1 \mathrm{~b}$ ), neither did additional excision or radiosurgery.

\section{Treatment of Recurrent CM}

No standard treatment has been established for patients with recurring brain metastases after RT. Reexcision, radiosurgery or reirradiation of the whole brain may be considered individually, as well as systemic treatment options. Retreatment seems reasonable only in cases with a progression-free interval of at least 4 months after initial treatment. The neurological symptoms, KPS, extracranial tumor control, and the desire of the patient are relevant criteria for the treatment decision (LoE 4). 
In some patients, systemic chemotherapy or, in Her2-overexpressing tumors, the combination of lapatinib and capecitabine might be an option (LoE 2b B; AGO: +).

\section{$R T$ and Systemic Treatment Options in LC}

RT is an effective palliative treatment for LC. As tumor dissemination affects the whole cerebrospinal fluid compartment, basically the complete cranio-spinal axis should be regarded as target volume. However, cranio-spinal irradiation is generally not recommended as it is assumed to cause substantial myelotoxicity. Instead, besides WBRT, involvedfield RT of bulky disease or symptomatic regions is mostly recommended. Nevertheless, in defined cases such as multiple circumscript plaques or nodules, RT of the neuroaxis should be considered.

Chemotherapy after irradiation bears an increased risk of leukencephalopathy.

Regional treatment options in LC:

Intrathecal or intraventricular therapy

- methotrexate (MTX) 10-15 mg 2-3 ×/week ( \pm folinic acid rescue) (LoE 2b, B; AGO: ++)

- liposomal cytarabine $50 \mathrm{mg}$, q 2w (LoE 3b, C; AGO: ++)

- thiothepa (LoE 3b, C; AGO: +)

- steroids (LoE 4, D; AGO: \pm )

\section{Technique and Dose Prescription}

For WBRT, the CTV encompasses the cerebrum plus cerebellum and brain stem. In case of leptomeningeal manifestation and infratentorial metastases, the field should reach down to the caudal margin of the second vertebral body. For LC, it is important to include the meningeal space with the lamina cribrosa and basal cisterns. 3D planning is useful for optimizing the dose distribution. A total dose of $30 \mathrm{~Gy}$ in daily fractional doses of 3 Gy and 5 fractions per week is most commonly used. In patients with a predicted survival exceeding 12 months, reduction of the single fraction to 2 Gy may be preferable $(20 \times 2 \mathrm{~Gy})$ in order to reduce brain toxicity. In cases of a more limited prognosis, acceleration of treatment time and single dose may be an alternative $(5 \times 4 \mathrm{~Gy})$. These recommendations apply to both $\mathrm{CM}$ and LC.

Stereotactic radiosurgery: For stereotactic irradiation, the gross tumor volume in MRI is regarded as CTV; an additional safety margin of 1-2 $\mathrm{mm}$ for the planning target volume (PTV) is recommended, depending on reproducibility and immobilization technique. Single-dose treatment is suitable for lesions up to $3.5 \mathrm{~cm}$ of size. A tumor-encompassing dose of $20-25$ Gy ( $80-90 \%$ isodose) is recommended, provided no WBRT has recently been performed or is planned consecutively. For tumors with a volume of $>4 \mathrm{ml}$ (i.e. a diameter $>2 \mathrm{~cm}$ ), the reference dose should not exceed $18 \mathrm{~Gy}$. When combined with WBRT, the radiosurgical dose should be restricted to $18 \mathrm{~Gy}$, and in larger tumors to $15 \mathrm{~Gy}$. Dose prescription refers the $80-90 \%$ isodoses.

Fractionated stereotactic $R T$ is feasible for tumors $>2 \mathrm{~cm}$ and lesions in critical anatomic sites such as a cerebellum with increased risk of incarceration. Moreover, fractionation is preferable for metastases of the brain stem, to avoid late reactions with dismal outcome. Depending on the treatment volume, fractionation schedules of $4 \times 8.7 \mathrm{~Gy}, 5 \times 7 \mathrm{~Gy}$, $6 \times 5$ Gy or $10 \times 4$ Gy are in use. In case of additional WBRT, $6 \times 5 \mathrm{~Gy}$ is recommended. Dose specification refers to the International Commission on Radiation Units and Measurements (ICRU) Report 50.

\section{Conflict of Interest}

This article has not been funded by any party. All authors confirm that they do not have any conflict of interest.

\section{References}

1 Souchon R, Wenz F, Sedlmayer F, et al.: DEGRO practice guidelines for palliative radiotherapy of metastatic breast cancer: Bone metastases and metastatic spinal cord compression (MSCC). Strahlenther Onkol 2009;185:417-424.

$\checkmark 2$ Feyer P, Sautter-Bihl ML, Budach W, et al.: DEGRO practical guidelines for palliative radiotherapy of breast cancer patients: Brain metastases and leptomeningeal carcinomatosis. Strahlenther Onkol 2010;186:63-69.

3 AGO Breast Committee: Diagnosis and treatment of patients with primary and metastatic breast cancer. Recommendations 2010. Version 1.1. www. ago-online.org.
Sze WM, Shelley M, Held I, et al.: Palliation of metastatic bone pain: single fraction versus multifraction radiotherapy - a systematic review of randomised trials. Clin Oncol (R Coll Radiol) 2003; 15:345-352.

5 Chow E, Harris K, Fan G, Tsao M, Sze WM: Palliative radiotherapy trials for bone metastases: a systematic review. J Clin Oncol 2007;25:1423-1436. 6 Foro Arnalot PF, Fontanals AV, Galceran JC, et al.: Randomized clinical trial with two palliative radiotherapy regimens in painful bone metastases: $30 \mathrm{~Gy}$ in 10 fractions compared with $8 \mathrm{~Gy}$ in single fraction. Radiother Oncol 2008;89:150-155.
Gaspar L, Scott Ch, Rotman M, et al.: Recursive partitioning analysis (RPA) of prognostic factors in three Radiation Therapy Oncology Group (RTOG) brain metastases trials. Int J Radiat Oncol Biol Phys 1997;37:745-751.

Gaspar L, Scott Ch, Murray K, et al.: Validation of the RTOG recursive partitioning analysis (RPA) classification for brain metastases. Int $\mathrm{J}$ Radiat Oncol Biol Phys 2000;47:1001-1006.

9 Rades D, Dunst J, Schild SE: A new scoring system to predicting the survival of patients treated with whole-brain radiotherapy for brain metastases. Strahlenther Onkol 2008;184:251-255. 